\title{
An Improved Method of Bronchial Stump Glosure for Prevention of Bronchopleural Fistula in Pulmonary Resection
}

\author{
Masami Sato, Yasuki Saito, Noriyoshi Nagamoto, \\ Chiaki Endo, Katuo Usuda, Satomi Takahashi, KeiJi \\ Kan'ma, Motoyasu Sagawa, Shin'ichiro Ota, Tasuku \\ NAKADA* and Shigefumi Fujimura \\ Department of Surgery, the Research Institute for \\ Tuberculosis and Cancer, Tohoku University, Sendai 980, \\ and ${ }^{*}$ Sendai Kohsei Hospital, Sendai 980
}

Sato, M., Saito, Y., Nagamoto, N., Endo C., Usuda, K., Takahashi, S., Kan'ma, K., Sagawa, M., Оhta, S., Nakada, T. and Fujimura, S. An Improved Method of Bronchial Stump Closure for Prevention of Bronchopleural Fistula in Pulmonary Resection. Tohoku J. Exp. Med., 1992, 168 (3), 507-513-We performed 880 pulmonary resections from January 1982 to June 1988 using Sweet's procedure for closure of the bronchial stump, in which 39 patients $(4.4 \%)$ developed bronchial fistulas. Bronchoscopic studies showed that bronchopleural fistualas were located mainly at the corner of the stump. This indicates that the corner is the point with the highest tension when Sweet's procedure is employed. In some cases, stumps were injured by suture materials, resulting in bronchopleural fistulas. Since July 1988, bronchial stumps have been closed by using two pairs of teflon pledgets with additional interrupted sutures. From July 1988 to April 1990, 288 patients were treated by this new method, and only one of them developed bronchopleural fistula. This new method prevents injury of the stump by suture material and reduces the tension at the bronchial stump for a long time. Thus, pulmonary resections can be safely employed even after anti-cancer chemotherapy and/or radiation therapy.— bronchopleural fistula; nonabsorbable suture; neoadjuvant therapy; teflon pledget

Postoperative bronchopleural fistula is a serious complication to treat once it has appeared. At present the incidence of such fistulas had remarkably decreased compared with that in the early history of thoracic surgery (Suzuki and Ueda 1955; Malave et al. 1971; Hankins et al. 1978). However, it has recently been reported that bronchopleural fistulas were detected in aged patients having complications and in patients who underwent completion pneumonectomy (Nishiwaki et al. 1989). Bronchopleural fistulas have also been detected in cases with pulmonary resection after neoadjuvant therapy (Ihde 1988) including radiation therapy or anticancer chemotherapy (Ohizumi et al. 1989 ; Moroboshi and Ishiba-

Received February 1, 1991 ; revision accepted for publication October 2, 1992 
shi 1989). Thus, the prevention of postoperative bronchopleural fistulas has again become urgent. Since July 1988, we have performed the bronchial stump closure using a new procedure with non-absorbable suture material as follows: two pairs of teflon pledgets are attached to the corners of the stump by horizontal mattress suturing, and some interrupted sutures are added for closure of the stump. We herein report that the incidence of postoperative bronchopleural fistulas has been remarkably decrease by this new method.

\section{Materials and Methods}

We examined the frequency of postoprative bronchopleural fistula in a total of 4286 patients who underwent pulmonary resection at the Research Institute for Tuberculosis and Cancer, Tohoku University between 1948 and April 1990. The method of Sweet (Sweet 1945) was used to close bronchial stumps until June 1988. Since July 1988, a new procedure of bronchial stump closure with two pairs of teflon pledgets had been employed instead of the Sweet's method. The effects of different stump closure methods and suture materials on the fistula frequency were compared in detail in 1168 cases of pulmonary resection for the period beginning in 1982 ( 880 cases with the Sweet's method, from January 1982 to June 1988, and 288 cases with the new method, from July 1988 to April 1990). For the same period starting in 1982, the frequencies of postoperative fistulas in bronchial stumps closed by Sweet's method and the new method were also compared in patients having inflammatory diseases and patients treated with neoadjuvant therapy. We also examined bronchopleural fistulas bronchoscopically in 26 cases in which stumps were closed by Sweet's method.

In Sweet's method, we used 3-0 silks until July 1985, 3-0 polyglactins (Vicryl; Ethicon Peterborough, Canada) from August 1985 to August 1987, and 2-0 polyglactins from September 1987 to June 1988. From July 1988 to April 1990, the new bronchial
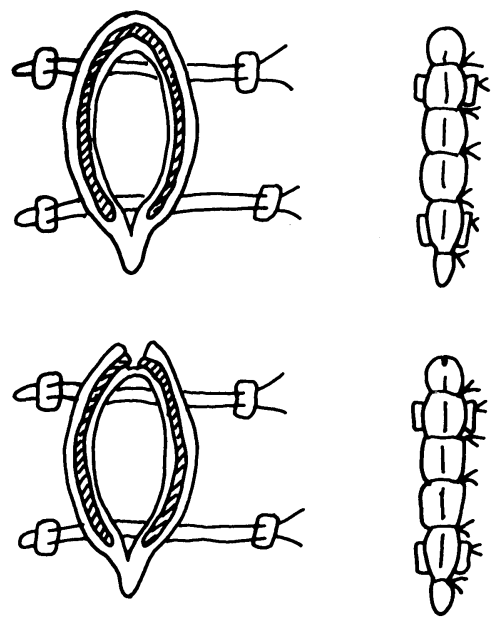

Fig. 1. Schema of bronchial stump closure. Two pairs of pledgets are attached by horizontal mattress suture, and a few interrupted sutures are employed on the stump. When tension is detected at the corner of the cartilage portion, the cartilage is cut (lower schema). Hatched areas represent cartilaginous portions. 

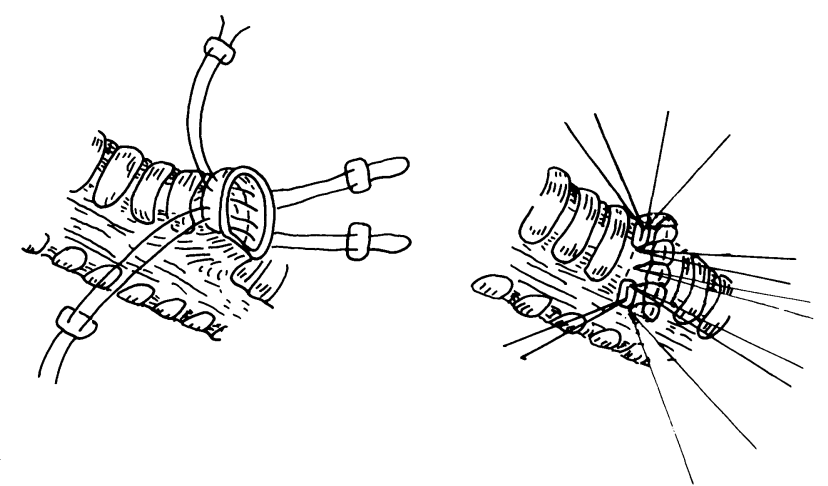

Fig. 2. Illustration of bronchial stump closure in case of right upper lobectomy.

stump closure method was employed using polyester sutures coated with polybutyrate (Ethibond ; Ethicon Peterborough, Canada) (Fig. 1 and 2). In this method, a pair of teflon pledgets were attached to each of the two corners of the stump with a horizontal mattress suture. And some interrupted sutures were added to close the stump. When the corner(s) of the cartilaginous portion had some tension, cartilage was cut at the corner(s) to reduce the tension. The stump was not covered in principle. The teflon pledgets used were $2.5 \mathrm{~mm} \times$ $6.6 \mathrm{~mm}$ and $1 \mathrm{~mm}$ thick. A 2-0 Ethibond suture was used for pneumonectomy, and a 3-0 Ethibond suture for lobectomy.

\section{RESUlts}

Postoperative bronchopleural fistulas were detected in $171(4.0 \%)$ of 4286 patients who underwent pulmonary resection between 1948 and April 1990 (Table 1). The incidence of bronchopleural fistulas was $131(4.2 \%)$ of 3118 cases of pulmonary resection between 1948 to 1981 (Table 1). All the cases of pulmonary resection performed between January 1982 and April 1990 were minutely examined, and postoperative bronchopleural fistulas were detected in $39(4.4 \%)$ of 880 cases in which bronchial stumps were closed by Sweet's method. Bronchoscopic findings indicated bronchopleural fistulas to be located at one or both of the corners of stumps in 19 of 26 fistula cases in which stumps were closed by Sweet's method (Table 2 ). In 3 of the 26 cases, fistulas seemed to have developed from injuries by suture material. Regarding the incidence in each of suture material groups, bronchopleural fistulas were detected in $21(4.6 \%)$ of 454 cases in which 3 0 silk was used, $14(5.3 \%)$ of 266 cases in which $3-0$ absorbable polyglactin was used, and $4(3.9 \%)$ of 102 cases in which $2-0$ absorbable polyglactin was used (Table 3). Neither absorbable material nor non-absorbable material had any influence on the frequencies, when Sweet's method was employed. On the other hand, in 288 cases of pulmonary resection which were subjected to the new stump closure procedure using teflon pledgets and polyester suture coated with polybutyrate, only one case showed bronchopleural fistula (Table 3). In patients who underwent pulmonary resection by Sweet's method for inflammatory diseases, 
TABLE 1. Incidence of postoperative bronchopleural fistulas

\begin{tabular}{|c|c|c|c|}
\hline Year & $\begin{array}{c}\text { Number of } \\
\text { pulmonary resection }\end{array}$ & $\begin{array}{c}\text { Bronchopleural fistula } \\
\text { (Number) }\end{array}$ & $(\%)$ \\
\hline $1948 \sim 1981$ & 3118 & 131 & 4.2 \\
\hline 1982 & 122 & 6 & 4.9 \\
\hline 1983 & 128 & 2 & 1.6 \\
\hline 1984 & 150 & 8 & 5.3 \\
\hline $1985^{\mathrm{a}}$ & 155 & 8 & 5.2 \\
\hline 1986 & 123 & 5 & 4.1 \\
\hline $1987^{\mathrm{b}}$ & 143 & 8 & 5.6 \\
\hline $1988^{\mathrm{b}, \mathrm{c}}$ & 143 & 2 & 1.4 \\
\hline Jan. $1982 \sim$ June $1988^{\mathrm{a}, \mathrm{b}}$ & 880 & 39 & 4.4 \\
\hline July $1988 \sim$ April $1990^{c}$ & 288 & 1 & 0.3 \\
\hline Total (1948 April 1990) & 4286 & 171 & 4.0 \\
\hline
\end{tabular}

${ }^{a}$ Before July 1985, 3-0 silk was used for stump closure. From August 1985 to August 1987, 3-0 polyglactin was used.

${ }^{b}$ From September 1987 to June 1988, 2-0 polyglactin was used.

cFrom July 1988 to April 1990, teflon pledgets and polybutyrate-coated polyester sutures were used.

TABLE 2. Bronchoscopic findings of bronchopleural fistulas in which stumps were closed by Sweet's method

\begin{tabular}{lc}
\hline Bronchoscopic findings & Number of cases \\
\hline Fully opened stump & 2 \\
Air leakage or bubbles at the corner(s) of the stump & 19 \\
a) at the corner beside the residual lobe & 10 \\
b) at the corner opposite to the residual lobe & 7 \\
c) at both corners of the stump & 2 \\
Rip in central portion of the stump & 3 \\
Effusion from peripheral bronchus & 2 \\
Total & 26 \\
\hline
\end{tabular}

bronchopleural fistulas were found in $4(7.7 \%)$ of the 52 patients in the $3-0$ silk group and in $1(6.3 \%)$ of the 16 patients in the polyglactin groups. However, no fistulas were observed in the cases in which teflon pledgets and polybutyratecoated polyester sutures were used (Table 4). In patients treated with preoperative neoadjuvant therapy including anticancer chemotherapy and radiation therapy, bronchopleural fistulas were detected in one of two cases in which the stumps were closed with polyglactin; however, fistulas were not detected in the cases in which teflon pledgets and polybutylate-coated polyester suture were used 
TABLE 3. Suture material and bronchopleural fistulas ${ }^{\mathrm{a}}$

\begin{tabular}{lccc}
\hline \multicolumn{1}{c}{ Suture material } & $\begin{array}{c}\text { Number of pulmonary } \\
\text { resections }\end{array}$ & $\begin{array}{c}\text { Bronchopleural fistulas } \\
\text { (Number) }\end{array}$ & $(\%)$ \\
\hline $3-0$ silk $^{\mathrm{b}}$ & 454 & 21 & 4.6 \\
$3-0$ polyglactin $^{\mathrm{b}}$ & 266 & 14 & 5.3 \\
2-0 polyglactin & $\mathrm{b}$ & 4 & 3.9 \\
Polyglactin (3-0+2-0) & 102 & 18 & 4.9 \\
Pledget+polyester & 368 & 1 & 0.3 \\
\hline
\end{tabular}

an this table, cases between 1982 and 1990 were included. Silk and polyglactin sutures were used in Sweet's method.

bVicryl

cPolyester suture coated with polybutyrate (Ethibond)

TABLE 4. Postoperative bronchopleural fistulas in patients with inflammatory diseases

\begin{tabular}{lcc}
\hline \multicolumn{1}{c}{ Suture material } & Number of resections & Number of fustulas \\
\hline 3-0 silk & 52 & $4(7.7 \%)$ \\
$3-0$ polyglactin & 13 & $1(1.1 \%)$ \\
2-0 polyglactin & 3 & 0 \\
Polyglactin $(3-0+2-0)$ & 16 & $1(6.3 \%)$ \\
Pledget + polyester & 10 & 0 \\
\hline
\end{tabular}

TABLE 5. Neoadjuvant therapy and bronchopleural fistulas

\begin{tabular}{ccc}
\hline Suture material & Number of patients & $\begin{array}{c}\text { Number of bronchopleural } \\
\text { fistulas }\end{array}$ \\
\hline Polyglactin & 2 & 1 \\
Pledget+polyester & 11 & 0 \\
\hline
\end{tabular}

(Table 5).

\section{Discussion}

During the period when surgical treatment was frequently employed to treat pulmonary tuberculosis, postoperative bronchopleural fistula was a common complication (Suzuki and Ueda 1955; Malave et al. 1971; Hankins et al. 1978). The incidence of such fistulas has remarkably decreased with the development and wide use of antituberculosis agents. Presently, the treatment of lung cancer, instead of tuberculosis, had become a major subject in the field of thoracic surgery. Many patients having lung cancer are aged heavy smokers, and most of them also have various complications. We think that risk factors for postoperative bronchopleural fistula have changed with the time. In addition, there are factors 
which arise from lung cancer itself, such as residual tumor on the stump, and problems caused by preoperative and postoperative anticancer chemotherapy or radiation therapy. Injuries of the bronchial artery and/or nerves in dissection of lymph nodes may also cause bronchopleural fistula (Wilkins 1987). All these factors should be examined as major causes of bronchopleural fistula (Brewer 1987). However, in general, studies of complications, as represented by bronchopleural fistula, are often affected by publishing bias ; i.e., studies with good results are apt to be published more often than those with bad results, and hence the literature represents an unrealistically favorable picture. Our previous reports revealed that brochopleural fistulas were found mostly in patients with residual cancer on the stump, in patients with advanced lung cancer, and in patients who had a poor pulmonary function and needed postoperative ventilatory support (Sato et al. 1989a, b). Some recent reports also showed that bronchopleural fistulas were frequently detected in patients who underwent completion pneumonectomy (Nishiwaki et al. 1989) and in those treated with neoadjuvant therapy (Ohizumi et al. 1989; Moroboshi and Ishibashi 1989). We think that postoperative bronchopleural fistulas is a problem which cannot be avoided when surgical treatment is actively applied to lung cancer.

Our previous findings on bronchoscopy indicated that the tension induced at the corners of the bronchial stump could cause bronchopleural fistulas (Sato et al. 1989a, b). In the present study we examined some additional cases of postoperative bronchopleural fistulas (Table 2). Bronchopleural fistulas were observed at one corner of the stump in most cases. In Sweet's method, the two portions of different lengths, i.e., cartilaginous portion and membranous portion, are sutured together. The end of the bronchial stump is usually cartilaginous, and the corners, but not the central part of the sutured stump, have high degree of tension. If the stump is not properly cured for some reason, such part has the highest probability of causing incomplete healing. In Sweet's method, absorbable and non-absorbable materials did not affect the frequency of postoperative bronchopleural fistulas (Table 3). The frequencies were around the level of $4 \%$ throughout the years in which Sweet's method had emplopyed (Table 1). This suggests that there are a few cases in which the bronchial stump cannot be closed by Sweet' s method. We consider that the tension induced at the corners of the stump is the main cause of postoperative fistulas. Bronchoscopic examination also revealed that cutting of the bronchial tissue was caused by suture material in 3 cases. Based on these two bronchoscopic findings, we decided to employ the new method using teflon pledgets attached to both the corners of the stump by horizontal mattress suturing. In this method, cartilaginous portions and membranous portions are sutured with some additional interrupted sutures for closing the stump. This method can prevent the cutting caused by closure material itself and results in a decrease in tension at the corner for a long time. It is also expected that pulmonary resection can be securely performed by this method. 
Recently, more and more cases of lung cancer have been treated with preoperative anticancer chemotherapy or radiation therapy. However, these therapies are apt to cause retarded healing and incomplete suture of the bronchial stump. This is a serious issue in neoadjuvant therapy; we indeed detected postoperative bronchopleural fistulas in patients treated with neoadjuvant therapy when we used the method of Sweet's method for closure of the bronchial stump. However, we have not detected any fistulas since we started using our new method for the closure. It is hence expected that the amount of preoperative radiation and the number of surgical cases can be increased in lung cancer, when the new method, which provides a secure bronchial stump closure, is used.

\section{References}

1) Brewer, L.A. (1987) Bronchopleural fistula: Management. In : International Trends in General Thoracic Surgery, Major Challenges, edited by H.C., Grillo \& H. Eschapasse, W.B. Saunders Company, Philadelphia, vol. 2, pp. 398-406.

2) Hankins, J.R., Miller, J.E., Attar, S., Satterfield, J.R. \& McLaughlin, J.S. (1978) Bronchopleural fistula: Thirteen-year experience with 77 cases. J. Thrac. Cardiovasc. Surg., 76, 755-762.

3) Ihde, D.C. (1988) Neoadjuvant chemotherapy for non-small cell lung cancer: Current North American experience, Semin. Oncol., 15, Suppl. 7, 3-7.

4) Malave, G., Foster, E.D., Wilson, J.A., \& Muro, D.D. (1971) Bronchopleural fistula, present-day study of an old problem: A review of 52 cases. Ann. Thrac. Surg., 11, 110.

5) Moroboshi, T. \& Ishibashi, M. (1989) A study of neoadjuvant therapy for advanced lung cancer. J. Jpn. Assoc. Chest Surg., 3, 251. (in Japanese)

6) Ohizumi, H., Kondo, H., Goya, A., Tsuchiya, R., Naruke, T. \& Shuematu K. (1989) An examination of cases with completion pneumonectomy. J. Jpn. Assoc. Chest Surg., 3, 256. (in Japanese)

7) Nishiwaki, Y., Nishiyama, Y. \& Kodama, T. (1989) Trial of neoadjuvant therapy focusing on cases at National Matsudo Hospital. In: The Forefront of Synthetical Treatment of Lung Cancer, edited by Ohta, M. \& Eguchi K., Gan to Kagakuryoho Sha, Tokyo, pp. 115-129. (in Japanese)

8) Sato, M., Saito, Y., Fujimura, S., Sagawa, M., Ohta, S., Nagamoto, N., Imai, T., Suda, S., Nakada, T. \& Hashimoto, K. (1989a) A study of recent postoperative bronchopleural fistula cases: Problems in diagnosis and results of reoperation in early stages. J. Jpn. Assoc. Thorac. Surg., 37, 11-19.

9) Sato, M., Saito, Y., Fujimura, S., Usuda, K., Takahashi, S., Kan'ma, K., Imai, T., Suda, S., Nakada, T. \& Hashimoto, K. (1989b) A study of recent postoperative bronchopleural fistula cases: Factors which may cause fistula. J. Jpn. Assoc. Thorac. Surg., 37, 487-503.

10) Suzuki, C. \& Ueda, H. (1955) Causes, diagnosis and treatment for bronchopleural fistulas after pulmonary resection. The lung, 2, 200-209. (in Japanese)

11) Sweet, R.H. (1945) Closure of the bronchial stump following lobectomy or pneumonectomy. Surgery, 18, 82-84.

12) Wilkins, E.W. (1987) Bronchopleural fistula: Prophylaxis. In : International Trends in General Thoracic Surgery, Major Challenges, edited by H.C., Grillo \& H. Eschapasse, W.B. Saunders Company, Philadelphia, vol 2, pp. 394-397. 